\title{
Nullities for a class of skew-symmetric Toeplitz band matrices
}

\author{
Ron Evans \\ Department of Mathematics \\ University of California at San Diego \\ La Jolla, CA 92093-0112 \\ revans@ucsd.edu \\ John Greene \\ Department of Mathematics and Statistics \\ University of Minnesota-Duluth \\ Duluth, MN 55812 \\ jgreene@d.umn.edu \\ Mark Van Veen \\ 2138 Edinburg Avenue \\ Cardiff by the Sea, CA 92007 \\ mavanveen@ucsd.edu
}

February 2020

2010 Mathematics Subject Classification. 15A03, 15A15, 15B05, 15B57, 05C20, 05C50

Key words and phrases. Toeplitz band matrix, skew-symmetric, graph cycles, nullity, matrix game, payoff matrix. 


\begin{abstract}
For all $n>k \geq 1$, we give formulas for the nullity $N(n, k)$ of the $n \times n$ skew-symmetric Toeplitz band matrix whose first $k$ superdiagonals have all entries 1 and whose remaining superdiagonals have all entries 0 . This is accomplished by counting the number of cycles in certain directed graphs. As an application, for each fixed integer $z \geq 0$ and large fixed $k$, we give an asymptotic formula for the percentage of $n>$ $k$ satisfying $N(n, k)=z$. For the purpose of rapid computation, an algorithm is devised that quickly computes $N(n, k)$ even for extremely large values of $n$ and $k$.
\end{abstract}

\title{
1 Introduction
}

For $n>k \geq 1$ and $x \in \mathbb{R}$, let $A(n, k, x)$ denote the $n \times n$ skew-symmetric Toeplitz matrix whose first $k$ superdiagonals have all entries 1 , and whose remaining superdiagonal entries are all $-x$. For example, $A(6,2, x)$ is the matrix

$$
\left[\begin{array}{cccccc}
0 & 1 & 1 & -x & -x & -x \\
-1 & 0 & 1 & 1 & -x & -x \\
-1 & -1 & 0 & 1 & 1 & -x \\
x & -1 & -1 & 0 & 1 & 1 \\
x & x & -1 & -1 & 0 & 1 \\
x & x & x & -1 & -1 & 0
\end{array}\right] .
$$

The matrices $A(n, k, x)$ are payoff matrices for the integer choice matrix games discussed for example in [1, 2, 3]. Let $N(n, k)$ denote the nullity of the skew-symmetric Toeplitz band matrix $A(n, k):=A(n, k, 0)$. The primary goal of this paper is to determine the nullities $N(n, k)$ for all $n>k \geq 1$. Our methods are different from those of Price et al. [5, 6], who investigated nullity sequences of Toeplitz matrices over finite fields. We say more about the benefit of our approach in the second paragraph of Section 6 .

There is an intriguing connection between the nullity $N(n, k)$ and the payoff matrix $A(n, k, x)$ when $n$ is even. Let $D(n, k, x) \in \mathbb{Z}[x]$ denote the determinant of $A(n, k, x)$. When $n$ is even, we conjecture that the zero $x=0$ of $D(n, k, x)$ has multiplicity $N(n, k)$. For example, the term of smallest degree in $D(16,4, x)$ is $81 x^{4}$, and $N(16,4)=4$. (If $n$ is odd, then of course the polynomial $D(n, k, x)$ vanishes identically, since the transpose of $A(n, k, x)$ equals $-A(n, k, x)$.) 
In Section 2, for each pair of integers $n, k$ with $k \geq 1$, we construct a directed graph $G(n, k)$ on the vertices $0,1, \ldots, k$ which consists of a disjoint union of pure cycles and an open path called a "tail". For example, $G(16,8)$ consists of the two cycles

$$
7 \rightarrow 1 \rightarrow 4 \rightarrow 7, \quad 6 \rightarrow 0 \rightarrow 3 \rightarrow 6
$$

together with the tail $8 \rightarrow 2 \rightarrow 5$. Theorem 2.1 shows that $N(n, k)$ equals the number of cycles in $G(n, k)$ (so for example $N(16,8)=2$ ). As shown at the end of Section 2, Theorem 2.1 enables one to rapidly compute the nullity $N(n, k)$ using Mathematica, even for extremely large $n, k$.

In order to obtain formulas for $N(n, k)$, we undertake a detailed analysis of the structure of the graph $G(n, k)$ in Sections 3-6, for each fixed $k$. Theorem 3.9 , the main result in Section 3, proves that for any fixed pair $k, n$, the cycles in $G(n, k)$ are "translates" of each other. In particular, these cycles all have the same length. Section 4 focuses on properties of the tail of $G(n, k)$. In Section 5, we prove lemmas that show how the number of cycles in $G(n, k)$ changes as $n$ increases. These results are then applied in Section 6 to describe the shape of the line graph connecting the points

$$
(n, N(n, k)), \quad 0 \leq n \leq k^{2}+k .
$$

Theorem 7.5, our main result, determines each location $n$ for which the nullity $N(n, k)$ has a local maximum in our line graph. We can then exploit the shape of the line graph to determine the values of all nullities $N(n, k)$, as is described at the beginning of Section 7.

Applications and examples are given in Section 8. For instance, Theorems 8.1 and 8.2 show that for large fixed $k$, about $30.4 \%$ of the matrices $A(n, k)$ are nonsingular, about $38 \%$ have nullity 1 , and about $11 \%$ have nullity 2 . The percentages continue to decrease as the nullities increase further.

In Theorem 9.2, we prove the aforementioned conjecture on the determinant $D(n, k, x)$ for all $k \geq(n-2) / 2$. The conjecture remains open in general for $k \leq(n-4) / 2$.

\section{The directed graph $G(n, k)$}

Let $\zeta$ be a complex primitive $k$-th root of unity and let $\xi$ be a complex primitive $(k+1)$-th root of unity. For integers $\ell$, define the $1 \times k$ row vectors

$$
u(\ell)=\left(1, \zeta^{\ell}, \zeta^{2 \ell}, \ldots, \zeta^{(k-1) \ell}\right), \quad v(\ell)=\left(\xi^{\ell}, \xi^{2 \ell}, \ldots, \xi^{k \ell}\right) .
$$


Throughout this paper, when $k$ or $k+1$ is explicitly employed as a subscript on an integer $\ell$, define $\ell_{k}$ (resp. $\ell_{k+1}$ ) to be the least nonnegative residue of $\ell$ modulo $k$ (resp. $k+1)$. Note that $u(\ell)=u\left(\ell_{k}\right)$ and $v(\ell)=v\left(\ell_{k+1}\right)$.

Define the $2 k \times 2 k$ matrix

$$
V(n, k)=\left(\begin{array}{c|c}
u(0) & v(0) \\
\vdots & \vdots \\
u(k-1) & v(k-1) \\
\hline u(k+n) & v(k+n) \\
\vdots & \vdots \\
u(2 k-1+n) & v(2 k-1+n)
\end{array}\right)=\left(\begin{array}{c|c}
A & B \\
\hline C & D
\end{array}\right)
$$

where $A, B, C, D$ are $k \times k$ matrices. Note that $V(0, k)$ is a Vandermonde matrix with distinct columns, so that its rows are independent over $\mathbb{C}$.

Converting the notation in [8] to ours, we see that our matrix $V(n, k)$ is the matrix in [8, eq. (6)]. Thus by [8, eq. (14)], $N(n, k)$ equals the nullity of $V(n, k)$. Consequently, for fixed $k, N(n, k)$ depends only on the value of $n$ $\left(\bmod k^{2}+k\right)$. We now drop the condition $n>k$ and extend the definition of $N(n, k)$ so that it equals the nullity of $V(n, k)$ for all integers $n$. In particular, $N(0, k)=0$.

The rows of $C$ in (2.1) are a cyclic permutation of the rows of $A$. Thus after row reduction, $V(n, k)$ can be converted to $\left(\begin{array}{c|c}A & B \\ \hline 0 & H\end{array}\right)$, where the $i$-th row of the $k \times k$ matrix $H$ is

$$
v\left((n+k+i-1)_{k+1}\right)-v\left((n+k+i-1)_{k}\right), \quad 1 \leq i \leq k .
$$

Since $H$ and $V(n, k)$ have the same nullity, $H$ has nullity $N(n, k)$. In particular, $N(n, k) \leq k$ for every $n$.

Define a directed graph $G(n, k)$ on the vertices $0,1, \ldots, k$ with edges $a \rightarrow b$ directed from $a$ to $b$ if and only if $v(a)-v(b)$ is a row in $H$. If $a=b$ we consider this edge to be a loop, i.e., a cycle of length 1 . In Theorem 2.1 below, we will show that the nullity of $H$ equals the number of cycles in the graph $G(n, k)$.

The graph $G(n, k)$ has exactly $k$ edges, namely

$$
E(i)=E(i, n):=(i+n-2)_{k+1} \rightarrow(i+n-1)_{k}, \quad 1 \leq i \leq k .
$$

Since no endpoint of an edge can be $k$, the vertex $k$ has in-degree 0 . Since no initial point of an edge can be $(n-2)_{k+1}$, the vertex $(n-2)_{k+1}$ has out-degree 
0 . For every other vertex, the in-degree and out-degree are both 1 . Thus $G(n, k)$ consists of an open path (called the "tail") connecting initial vertex $k$ to terminal vertex $(n-2)_{k+1}$, together with a (possibly empty) disjoint union of simple cycles. The number of edges in a path $P$ is the path length, denoted by $|P|$. If it happens that $k=(n-2)_{k+1}$, then the tail has length 0 and $k$ is an isolated vertex.

For a cycle $C$ in $G(n, k)$, let $R(C)$ denote the set of row positions in the matrix $H$ corresponding to the edges of $C$. For example, if $C$ is the cycle

$$
1 \rightarrow 4 \rightarrow 8 \rightarrow 5 \rightarrow 9 \rightarrow 1
$$

then $R(C)$ is the set of row positions of $v(1)-v(4), v(4)-v(8), v(8)-v(5)$, $v(5)-v(9), v(9)-v(1)$ in the matrix $H$. Observe that $C$ corresponds to a $1 \times k$ row vector of 0 's and 1 's in the left nullspace of $H$, where the 1 's are in the positions matching the row positions in $R(C)$.

Theorem 2.1. The nullity $N(n, k)$ of the matrix $H$ equals the number of cycles in the graph $G(n, k)$.

Proof. As noted above, each cycle corresponds to a row vector in the left nullspace $\mathcal{L}$ of $H$. Since the cycles are disjoint, the corresponding row vectors are independent over $\mathbb{C}$. These row vectors therefore span a subspace $\mathcal{A} \subset \mathcal{L}$ whose dimension equals the number of cycles. It remains to show that $\mathcal{A}=\mathcal{L}$.

Let $\mathcal{T}$ denote the set of all (nonzero) complex row vectors $\tau \in \mathcal{L}$ such that $\tau \notin \mathcal{A}$. Our goal is to show that $\mathcal{T}$ is empty.

Suppose for the purpose of contradiction that $\mathcal{T}$ contains a vector $\tau$. The product $\tau H$ is a linear combination of the rows of $H$ whose coefficients are the entries in $\tau$. This linear combination sums to 0 , since $\tau \in \mathcal{L}$.

For each cycle $C$, consider the $|C|$ entries in $\tau$ situated in the positions matching the row positions in $R(C)$. Replace one of these $|C|$ coefficients (call it $c$ ) by 0 and then subtract $c$ from each of the remaining $|C|-1$ coefficients. Since such replacements are made for every cycle $C$, this yields a new vector $\tau^{\prime} \in \mathcal{T}$ with the property that $\tau^{\prime} H$ is a linear combination of rows of $H$ corresponding to edges of disjoint open paths. For example, the subsum of $\tau^{\prime} H$ corresponding to an open path such as

$$
2 \rightarrow 8 \rightarrow 6 \rightarrow 3 \rightarrow 7
$$

would have the form

$$
d_{1}(v(2)-v(8))+d_{2}(v(8)-v(6))+d_{3}(v(6)-v(3))+d_{4}(v(3)-v(7)) .
$$


The only dependence relations among the vectors $v(0), \ldots, v(k)$ are

$$
\alpha(v(0)+v(1)+\cdots+v(k))=0, \quad \alpha \in \mathbb{C} .
$$

Since $\tau^{\prime} H=0$, the coefficients of the vectors $v(\ell)$ in the expansion of $\tau^{\prime} H$ must all be equal.

Let us first focus on the subsum in our example (2.3). Expanding in $\tau^{\prime} H$, this subsum becomes

$$
d_{1} v(2)+\left(d_{2}-d_{1}\right) v(8)+\left(d_{3}-d_{2}\right) v(6)+\left(d_{4}-d_{3}\right) v(3)-d_{4} v(7) \text {. }
$$

Thus

$$
d_{1}=d_{2}-d_{1}=d_{3}-d_{2}=d_{4}-d_{3}=-d_{4} .
$$

The five equal members of this equality sum to zero, so all five must vanish. Therefore the $d_{i}$ all vanish. This type of argument applies generally to each of the disjoint subsums of $\tau^{\prime} H$ corresponding to an open path, showing that all entries of $\tau^{\prime}$ vanish. This contradicts the fact that $\tau^{\prime} \in \mathcal{T}$.

Due to Theorem 2.1 , the nullity of $A(n, k)$ can be rapidly computed for very large values of the arguments $n, k$, using this function in Mathematica:

FastNullity $\left[\mathrm{n}_{-}, \mathrm{k}_{-}\right]:=$

Length [FindCycle $[$ Table $[\operatorname{Mod}[i-2+n, 1+k]->\operatorname{Mod}[i-1+n, k],\{i, 1, k\}]$

$, k, A l l]]+\operatorname{Sum}[\operatorname{If}[\operatorname{Mod}[i-2+n, 1+k]==\operatorname{Mod}[i-1+n, k], 1,0],\{i, 1, k\}]$

\section{Properties of the cycles in $G(n, k)$}

Fix a pair $n, k$. The object of this section is to prove Theorem 3.9, which shows that the cycles of $G(n, k)$ are "translates" of each other. A consequence of Theorem 3.9 is the nontrivial fact that the cycles have the same length. We begin with a string of eight lemmas.

Recall that when $k$ or $k+1$ is explicitly employed as a subscript on some integer $\beta$, we have defined $\beta_{k}$ (resp. $\beta_{k+1}$ ) to be the least nonnegative residue of $\beta$ modulo $k$ (resp. $k+1$ ).

Lemma 3.1. Suppose that

$$
a_{1} \rightarrow a_{2} \rightarrow \cdots \rightarrow a_{\ell}
$$


is a path in $G(n, k)$. Then

$$
\left(a_{1}+1\right)_{k+1} \rightarrow \cdots \rightarrow\left(a_{\ell}+1\right)_{k+1}
$$

is a path in $G(n, k)$ if and only if none of $a_{2}, \ldots, a_{\ell}$ equals $k-1$ and none of $a_{1}, \ldots, a_{\ell-1}$ equals $(n-3)_{k+1}$.

Proof. Suppose that (3.2) holds. Then none of $a_{2}, \ldots, a_{\ell}$ can equal $k-1$, since $k$ has in-degree 0 , and none of $a_{1}, \ldots, a_{\ell-1}$ can equal $(n-3)_{k+1}$, since $(n-2)_{k+1}$ has out-degree 0 .

Conversely, if none of $a_{1}, \ldots, a_{\ell-1}$ equals $(n-3)_{k+1}$, then by (2.2), we have $i<k$ for any edge $E(i):=a_{\nu} \rightarrow a_{\nu+1}$ in the path (3.1). If moreover none of $a_{2}, \ldots, a_{\ell}$ equals $k-1$, then $E(i+1)$ is the edge $\left(a_{\nu}+1\right)_{k+1} \rightarrow\left(a_{\nu+1}+1\right)_{k+1}$, so (3.2) follows.

Lemma 3.2. Suppose that

$$
a_{1} \rightarrow \cdots \rightarrow a_{\ell-1} \rightarrow k-1
$$

is a path in $G(n, k)$. Then

$$
\left(a_{1}+1\right)_{k+1} \rightarrow \cdots \rightarrow\left(a_{\ell-1}+1\right)_{k+1} \rightarrow 0
$$

is a path in $G(n, k)$ if and only if none of $a_{1}, \ldots, a_{\ell-1}$ equals $(n-3)_{k+1}$.

Proof. If none of $a_{1}, \ldots, a_{\ell-1}$ equals $(n-3)_{k+1}$, then $i<k$ for the edge

$$
E(i):=a_{\ell-1} \rightarrow k-1=(i+n-1)_{k},
$$

so that $E(i+1)$ is the edge

$$
\left(a_{\ell-1}+1\right)_{k+1} \rightarrow(i+n)_{k}=0 .
$$

Lemma 3.3. Suppose that

$$
a_{1} \rightarrow a_{2} \rightarrow \cdots \rightarrow a_{\ell}
$$

is a path in $G(n, k)$. Then

$$
\left(a_{1}-1\right)_{k+1} \rightarrow \cdots \rightarrow\left(a_{\ell}-1\right)_{k+1}
$$

is a path in $G(n, k)$ if and only if none of $a_{2}, \ldots, a_{\ell}$ equals 0 and none of $a_{1}, \ldots, a_{\ell-1}$ equals $(n-1)_{k+1}$. 
Proof. Suppose that (3.6) holds. Then none of $a_{2}, \ldots, a_{\ell}$ can equal 0 , since $k$ has in-degree 0 , and none of $a_{1}, \ldots, a_{\ell-1}$ can equal $(n-1)_{k+1}$, since $(n-2)_{k+1}$ has out-degree 0 .

Conversely, if none of $a_{1}, \ldots, a_{\ell-1}$ equals $(n-1)_{k+1}$, then by (2.2), we have $i>1$ for any edge $E(i):=a_{\nu} \rightarrow a_{\nu+1}$ in the path (3.5). If moreover none of $a_{2}, \ldots, a_{\ell}$ equals 0 , then $E(i-1)$ is the edge $\left(a_{\nu}-1\right)_{k+1} \rightarrow\left(a_{\nu+1}-1\right)_{k+1}$, so (3.6) follows.

Lemma 3.4. Suppose that

$$
a_{1} \rightarrow \cdots \rightarrow a_{\ell-1} \rightarrow 0
$$

is a path in $G(n, k)$. Then

$$
\left(a_{1}-1\right)_{k+1} \rightarrow \cdots \rightarrow\left(a_{\ell-1}-1\right)_{k+1} \rightarrow k-1
$$

is a path in $G(n, k)$ if and only if none of $a_{1}, \ldots, a_{\ell-1}$ equals $(n-1)_{k+1}$.

Proof. If none of $a_{1}, \ldots, a_{\ell-1}$ equals $(n-1)_{k+1}$, then $i>1$ for the edge

$$
E(i):=a_{\ell-1} \rightarrow 0=(i+n-1)_{k},
$$

so that $E(i-1)$ is the edge

$$
\left(a_{\ell-1}-1\right)_{k+1} \rightarrow(i+n-2)_{k}=k-1 .
$$

Let $C$ denote a cycle in $G(n, k)$ of the form

$$
a_{1} \rightarrow \cdots \rightarrow a_{\ell} \rightarrow a_{1} .
$$

If for some integer $t, G(n, k)$ has a cycle

$$
\left(a_{1}+t\right)_{k+1} \rightarrow \cdots \rightarrow\left(a_{\ell}+t\right)_{k+1} \rightarrow\left(a_{1}+t\right)_{k+1},
$$

denote this cycle by $C+t$. We refer to $C+t$ as a "translate" of $C$.

Lemma 3.5. For the cycle $C$ in (3.9), $C+1$ is a cycle if and only if $C$ avoids the vertices $k-1$ and $(n-3)_{k+1}$, and $C-1$ is a cycle if and only if $C$ avoids the vertices 0 and $(n-1)_{k+1}$. 
Proof. This follows from Lemmas 3.1 and 3.3.

Lemma 3.6. For the cycle $C$ in (3.9), $C+t$ is a cycle for each $t \in[-v, u]$, where $u \geq 0$ is minimal for which at least one of $k-1,(n-3)_{k+1}$ is a vertex in $C+u$, and $v \geq 0$ is minimal for which at least one of $0,(n-1)_{k+1}$ is a vertex in $C-v$.

Proof. If $C$ avoids vertices $k-1$ and $(n-3)_{k+1}$, then $C+1$ is a cycle by Lemma 3.5. The translation by 1 can be interated but it cannot go on indefinitely, otherwise given any vertex $a \in C$, the translate $C+(k-a)$ would be a cycle containing $k$, a contradiction. Thus the iteration must eventually reach the cycle $C+u$, after which the process stops, by Lemma 3.5. Similarly, translation by -1 can be iterated, with the process stopping upon reaching the cycle $C-v$.

Lemma 3.7. For $G(n, k)$, the following statements are equivalent:

(A) There are no cycles.

(B) Both $k-1$ and $(n-3)_{k+1}$ lie in the tail.

(C) Both 0 and $(n-1)_{k+1}$ lie in the tail.

(D) Both 0 and $k-1$ lie in the tail.

Proof. Clearly (A) implies (B), (C), and (D).

Conversely, suppose that $(\mathrm{A})$ is false, so that there exists a cycle $C$. Then by Lemma 3.6, at least one of $k-1,(n-3)_{k+1}$ lies in $C+u$, and at least one of $0,(n-1)_{k+1}$ lies in $C-v$, so $(\mathrm{B})$ and $(\mathrm{C})$ are false. It remains to prove that (D) is false. Assuming that $k-1$ lies in the tail, we must show that 0 lies in a cycle. The tail must have an initial segment of the form

$$
k \rightarrow a_{2} \rightarrow \cdots \rightarrow a_{\ell-1} \rightarrow k-1 .
$$

Since $(\mathrm{B})$ is false, $(n-3)_{k+1}$ does not lie in the tail. Thus by Lemma 3.2, $G(n, k)$ has the cycle

$$
0 \rightarrow\left(a_{2}+1\right)_{k+1} \rightarrow \cdots \rightarrow\left(a_{\ell-1}+1\right)_{k+1} \rightarrow 0 .
$$

Lemma 3.8. Suppose that neither 0 nor $k-1$ lies in the tail of $G(n, k)$. Then $G(n, k)$ has a cycle $C^{\prime}$ containing both $k-1$ and $(n-3)_{k+1}$ and a cycle $C^{\prime \prime}$ containing both 0 and $(n-1)_{k+1}$. Moreover, $C^{\prime}$ is longer than the tail. 
Proof. The cycle containing $k-1$ has the form

$$
k-1 \rightarrow a_{2} \rightarrow \cdots \rightarrow a_{\ell-1} \rightarrow k-1 .
$$

It must be that $(n-3)_{k+1}$ lies in this cycle, for otherwise, by Lemma 3.2, the tail would begin

$$
k \rightarrow\left(a_{2}+1\right)_{k+1} \rightarrow \cdots \rightarrow\left(a_{\ell-1}+1\right)_{k+1} \rightarrow 0,
$$

which contradicts the fact that 0 is not in the tail.

To see that this cycle is longer than the tail, note that if $(n-3)_{k+1}=a_{i}$, then the full tail is

$$
k \rightarrow\left(a_{2}+1\right)_{k+1} \rightarrow \cdots \rightarrow\left(a_{i}+1\right)_{k+1}=(n-2)_{k+1},
$$

which is shorter than the cycle. If on the other hand $(n-3)_{k+1}=k-1$, then $(n-2)_{k+1}=k$, wherein the tail has length 0 .

We now consider the cycle containing 0 . For some $\ell$, this cycle has the form

$$
0 \rightarrow a_{2} \rightarrow \cdots \rightarrow a_{\ell-1} \rightarrow 0 .
$$

It must be that $(n-1)_{k+1}$ lies in this cycle, for otherwise, by Lemma 3.4, the tail would begin

$$
k \rightarrow\left(a_{2}-1\right)_{k+1} \rightarrow \cdots \rightarrow\left(a_{\ell-1}-1\right)_{k+1} \rightarrow k-1,
$$

which contradicts the fact that $k-1$ is not in the tail.

Theorem 3.9. For any fixed pair $k, n$, all cycles in $G(n, k)$ are contiguous translates of each other. In particular, these cycles have the same length.

Proof. Let $C_{1}$ and $C_{2}$ be two different cycles in $G(n, k)$. We will consider three cases: when 0 is in the tail, when $k-1$ is in the tail, and when neither 0 nor $k-1$ is in the tail.

First suppose that $k-1$ lies in the tail. By Lemma 3.6, there are nonnegative integers $u_{1}$ and $u_{2}$ for which the translates $C_{1}+u_{1}$ and $C_{2}+u_{2}$ both contain $(n-3)_{k+1}$, while $C_{1}+\alpha$ is a cycle for all $0 \leq \alpha \leq u_{1}$ and $C_{2}+\beta$ is a cycle for all $0 \leq \beta \leq u_{2}$. Since $C_{1}+u_{1}$ and $C_{2}+u_{2}$ are not disjoint, we have $C_{1}+u_{1}=C_{2}+u_{2}$, so that $C_{2}=C_{1}+\left(u_{1}-u_{2}\right)$. Without loss of generality, $u_{1} \geq u_{2}$. Thus $C_{2}$ is one of the contiguous cycles $C_{1}+\alpha$ listed above. 
Next suppose that 0 lies in the tail. By Lemma 3.6, there are nonnegative integers $v_{1}$ and $v_{2}$ for which the translates $C_{1}-v_{1}$ and $C_{2}-v_{2}$ both contain $(n-1)_{k+1}$, while $C_{1}-\alpha$ is a cycle for all $0 \leq \alpha \leq v_{1}$ and $C_{2}-\beta$ is a cycle for all $0 \leq \beta \leq v_{2}$. Since $C_{1}-v_{1}$ and $C_{2}-v_{2}$ are not disjoint, we have $C_{1}-v_{1}=C_{2}-v_{2}$, so that $C_{2}=C_{1}-\left(v_{1}-v_{2}\right)$. Without loss of generality, $v_{1} \geq v_{2}$. Thus $C_{2}$ is one of the contiguous cycles $C_{1}-\alpha$ listed above.

Finally suppose that neither 0 nor $k-1$ lies in the tail. By Lemma 3.6 and Lemma 3.8, there exist nonnegative integers $u_{1}$ and $u_{2}$ for which the translates $C_{1}+u_{1}$ and $C_{2}+u_{2}$ are both equal to the cycle $C^{\prime}$ defined in Lemma 3.8, while $C_{1}+\alpha$ is a cycle for all $0 \leq \alpha \leq u_{1}$ and $C_{2}+\beta$ is a cycle for all $0 \leq \beta \leq u_{2}$. Thus, $C_{2}=C_{1}+\left(u_{1}-u_{2}\right)$, where without loss of generality, $u_{1} \geq u_{2}$. Thus $C_{2}$ is one of the contiguous cycles $C_{1}+\alpha$ listed above.

\section{Properties of the tail in $G(n, k)$}

Lemma 4.1. Let $C$ be any cycle in $G(n, k)$. If 0 or $k-1$ is in the tail of $G(n, k)$, then the tail is at least as long as $C$.

Proof. First suppose that $k-1$ is in the tail. Then the tail has an initial segment of the form

$$
k \rightarrow a_{2} \rightarrow \cdots \rightarrow a_{\ell-1} \rightarrow k-1 .
$$

This path has length $\ell-1$. By Lemma 3.7, $(n-3)_{k+1}$ is not in the tail, so by Lemma $3.2, G(n, k)$ has the following cycle of length $\ell-1$ :

$$
0 \rightarrow\left(a_{2}+1\right)_{k+1} \rightarrow \cdots \rightarrow\left(a_{\ell-1}+1\right)_{k+1} \rightarrow 0 .
$$

Since all cycles have the same length by Theorem 3.9, the tail is at least as long as $C$.

Now suppose that 0 is in the tail. Then for some $\ell$, the tail has an initial segment of the form

$$
k \rightarrow a_{2} \rightarrow \cdots \rightarrow a_{\ell-1} \rightarrow 0 .
$$

This path has length $\ell-1$. By Lemma 3.7, $(n-1)_{k+1}$ is not in the tail, so by Lemma 3.4, $G(n, k)$ has the following cycle of length $\ell-1$ :

$$
k-1 \rightarrow\left(a_{2}-1\right)_{k+1} \rightarrow \cdots \rightarrow\left(a_{\ell-1}-1\right)_{k+1} \rightarrow k-1 .
$$

Thus again, the tail is at least as long as $C$. 
Lemma 4.2. Suppose that neither 0 nor $k-1$ lies in the tail of $G(n, k)$. Let $q$ denote the lengths of the cycles $C^{\prime}$ and $C^{\prime \prime}$ defined in Lemma 3.8. Write $k=f q+m$, where $f$ is the floor $[k / q]$ and $0 \leq m<q$. Then $G(n, k)$ consists of the $f$ cycles

$$
C^{\prime}, C^{\prime}-1, \ldots, C^{\prime}-(f-1)
$$

together with a tail $\left(C^{\prime}+1\right)^{*}$ of length $m$, where $\left(C^{\prime}+1\right)^{*}$ denotes the segment of $\left(C^{\prime}+1\right)$ beginning with $k$ and ending with $(n-2)_{k+1}$. At the same time, $G(n, k)$ consists of the $f$ cycles

$$
C^{\prime \prime}, C^{\prime \prime}+1, \ldots, C^{\prime \prime}+(f-1)
$$

together with a tail $\left(C^{\prime \prime}-1\right)^{*}$ of length $m$, where $\left(C^{\prime \prime}-1\right)^{*}$ denotes the segment of $\left(C^{\prime \prime}-1\right)$ beginning with $k$ and ending with $(n-2)_{k+1}$. As a consequence, $C^{\prime}=C^{\prime \prime}+(f-1)$ and $\left(C^{\prime}+1\right)^{*}=\left(C^{\prime \prime}-1\right)^{*}$.

Proof. Since all cycles have length $q$ and the tail has length $<q$ by Lemma 3.8 , there must be exactly $f$ cycles of length $q$ and a tail of length $m$. There is no cycle $\left(C^{\prime}+1\right)$, because $C^{\prime}$ contains $k-1$, and adding 1 to $k-1$ yields the vertex $k$ in the tail. Thus the set of cycles must consist of the $f$ contiguous cycles in (4.1). Similarly, since $C^{\prime \prime}$ contains 0 , there is no cycle $\left(C^{\prime \prime}-1\right)$, so the set of cycles must consist of the $f$ contiguous cycles in (4.2). By Lemma 3.8 , the path

$$
k-1 \rightarrow \cdots \rightarrow(n-3)_{k+1}
$$

is part of the cycle $C^{\prime}$, so the tail must be $\left(C^{\prime}+1\right)^{*}$. Similarly, the path

$$
0 \rightarrow \cdots \rightarrow(n-1)_{k+1}
$$

is part of the cycle $C^{\prime \prime}$, so the tail must be $\left(C^{\prime \prime}-1\right)^{*}$.

Define translates of paths exactly as we did for cycles. To reiterate, suppose $D$ is a path in $G(n, k)$ of the form $d_{1} \rightarrow \cdots \rightarrow d_{\ell}$. If for some integer $t$, there is a path $\left(d_{1}+t\right)_{k+1} \rightarrow \cdots \rightarrow\left(d_{\ell}+t\right)_{k+1}$ in $G(n, k)$, we call this path the translate $D+t$.

Lemma 4.3. Suppose that $k-1$ is in the tail of $G(n, k)$ but $(n-3)_{k+1}$ is not. Thus, for some $\ell \geq 2$, the tail must have an initial segment of length $\ell-1$ given by

$$
P \rightarrow k-1
$$


where $P$ is an open path of length $\ell-2$ of the form

$$
k \rightarrow a_{2} \rightarrow \cdots \rightarrow a_{\ell-1} .
$$

Let $C$ denote the cycle of length $\ell-1$ given by

$$
0 \rightarrow\left(a_{2}+1\right)_{k+1} \rightarrow \cdots \rightarrow\left(a_{\ell-1}+1\right)_{k+1} \rightarrow 0,
$$

which exists by Lemma 3.2. Then $G(n, k)$ consists of the set of cycles

$$
C, C+1, \ldots, C+u,
$$

with $u$ as defined in Theorem 3.6, together with a tail of the form

$$
P \rightarrow(P-1) \rightarrow \cdots \rightarrow(P-t) \rightarrow(P-t-1)^{*},
$$

where $t \geq 0$ is minimal for which $P-t$ contains $(n-1)_{k+1}$, and $(P-t-1)^{*}$ denotes the initial segment of $(P-t-1)$ which terminates with the vertex $(n-2)_{k+1}$.

Proof. Since $C$ contains 0 , there is no cycle $C-1$, so by Theorem 3.9, the contiguous cycles in (4.4) must be the full set of cycles in $G(n, k)$. By (4.3) and Lemma 3.3, the tail begins

$$
P \rightarrow(P-1) \rightarrow \cdots \rightarrow(P-t) .
$$

Finally, since $(P-t)$ contains $(n-1)_{k+1}$ and $\left((n-1)_{k+1}-1\right)_{k+1}$ equals the terminal vertex $(n-2)_{k+1}$, we have $(P-t) \rightarrow(P-t-1)^{*}$.

Lemma 4.4. Suppose that 0 is in the tail of $G(n, k)$ but $(n-1)_{k+1}$ is not. Thus, for some $\ell \geq 2$, the tail must have an initial segment of length $\ell-1$ given by

$$
P \rightarrow 0
$$

where $P$ is an open path of length $\ell-2$ of the form

$$
k \rightarrow a_{2} \rightarrow \cdots \rightarrow a_{\ell-1} .
$$

Let $C$ denote the cycle of length $\ell-1$ given by

$$
k-1 \rightarrow\left(a_{2}-1\right)_{k+1} \rightarrow \cdots \rightarrow\left(a_{\ell-1}-1\right)_{k+1} \rightarrow k-1,
$$


which exists by Lemma 3.4. Then $G(n, k)$ consists of the set of cycles

$$
C, C-1, \ldots, C-v \text {, }
$$

with $v$ as defined in Theorem 3.6, together with a tail of the form

$$
P \rightarrow(P+1) \rightarrow \cdots \rightarrow(P+t) \rightarrow(P+t+1)^{*},
$$

where $t \geq 0$ is minimal for which $P+t$ contains $(n-3)_{k+1}$, and $(P+t+1)^{*}$ denotes the initial segment of $(P+t+1)$ which terminates with the vertex $(n-2)_{k+1}$.

Proof. Since $C$ contains $k-1$, there is no cycle $C+1$, so by Theorem 3.9, the contiguous cycles in (4.7) must be the full set of cycles in $G(n, k)$. By (4.6) and Lemma 3.1, the tail begins

$$
P \rightarrow(P+1) \rightarrow \cdots \rightarrow(P+t)
$$

Finally, since $(P+t)$ contains $(n-3)_{k+1}$ and $\left((n-3)_{k+1}+1\right)_{k+1}$ equals the terminal vertex $(n-2)_{k+1}$, we have $(P+t) \rightarrow(P+t+1)^{*}$.

Lemma 4.5. For the graph $G(n, k)$, the following are equivalent:

(A) $k-1$ is in the tail.

(B) $n_{k}$ is in the tail.

(C) $(n-1)_{k+1}$ is in the tail.

Proof. By (2.2) with $i=1,(\mathrm{~B})$ and (C) are equivalent, so it remains to prove that $(\mathrm{A})$ is equivalent to $(\mathrm{C})$. The result is obvious if there are no cycles, so assume there is a cycle $C$.

First assume (A). By Lemma 3.7, $(n-3)_{k+1}$ cannot lie in the tail, so that the hypotheses of Lemma 4.3 hold. Now (C) follows, since the segment $P-t$ in (4.5) contains $(n-1)_{k+1}$.

Conversely, assume that (A) is false. We must show that $(n-1)_{k+1}$ lies in a cycle. If 0 is not in the tail, then $(n-1)_{k+1}$ lies in the cycle $C^{\prime \prime}$, by Lemma 3.8. If 0 lies in the tail, then $(n-1)_{k+1}$ lies in the cycle $C-v$, where $v$ is defined in Lemma 3.6.

\section{Relation between $G(n, k)$ and $G(n+1, k)$}

Recall from (2.2) that $G(n, k)$ has the $k$ edges

$$
E(i, n):=(i+n-2)_{k+1} \rightarrow(i+n-1)_{k}, \quad 1 \leq i \leq k .
$$

The following lemma relates these to the edges of $G(n+1, k)$. 
Lemma 5.1. The $k$ edges of $G(n+1, k)$ consist of the $k-1$ edges

$$
E(2, n), E(3, n), \ldots, E(k, n)
$$

from $G(n, k)$ together with the additional edge $E(k, n+1)$. Thus the only edge in $G(n, k)$ which is not also an edge in $G(n+1, k)$ is

$$
E(1, n):=(n-1)_{k+1} \rightarrow n_{k}
$$

this edge is "replaced" in $G(n+1, k)$ by

$$
E(k, n+1):=(n-2)_{k+1} \rightarrow n_{k} .
$$

Proof. The result follows because

$$
E(i+1, n)=E(i, n+1), \quad 1 \leq i \leq k-1 .
$$

Lemma 5.2. If $C$ is a cycle in $G(n, k)$ which does not contain $n_{k}$, then $C$ remains a cycle in $G(n+1, k)$.

Proof. By Lemma 5.1, all the edges in $C$ remain edges in $G(n+1, k)$, since $E(1, n)$ is not an edge in $C$.

Lemma 5.3. Suppose that $G(n, k)$ has a cycle $C$ given by

$$
a_{1} \rightarrow a_{2} \rightarrow \cdots \rightarrow a_{\ell} \rightarrow a_{1}
$$

with $a_{1}=n_{k}$. Then the tail in $G(n+1, k)$ is

$$
T \rightarrow a_{1} \rightarrow \cdots \rightarrow a_{\ell}
$$

where $T$ is the tail

$$
T:=k \rightarrow \cdots \rightarrow(n-2)_{k+1}
$$

in $G(n, k)$.

Proof. The last edge in (5.1) is $E(1, n)$, since $a_{1}=n_{k}$. Thus $a_{\ell}$ is the terminal vertex $(n-1)_{k+1}$ in $G(n+1, k)$. Since $E(k, n+1)$ is an edge in $G(n+1, k)$, we see that $T \rightarrow a_{1}$ in $G(n+1, k)$. Finally, the edges

$$
a_{i} \rightarrow a_{i+1}, \quad 1 \leq i \leq \ell-1
$$

in $G(n, k)$ all lie in $G(n+1, k)$ by Lemma 5.1 . 
We say that $G(n, k)$ 's tail $T$ in (5.3) will absorb $G(n, k)$ 's cycle $C$ in (5.1) if (5.2) gives the tail in $G(n+1, k)$.

Lemma 5.4. If $k-1$ is not in $G(n, k)$ 's tail $T$, then $T$ will absorb a cycle.

Proof. The result follows from Lemma 5.3 and Lemma 4.5.

Lemma 5.5. Suppose that $n_{k}$ lies in the tail $T$ of $G(n, k)$, so that $T$ must have the form

$$
T:=T_{1} \rightarrow n_{k} \rightarrow \cdots \rightarrow(n-2)_{k+1},
$$

where $T_{1}$ is the tail in $G(n+1, k)$ given by

$$
T_{1}:=k \rightarrow \cdots \rightarrow(n-1)_{k+1} .
$$

Then the cycles in $G(n, k)$ remain cycles in $G(n+1, k)$, and $G(n+1, k)$ has one additional cycle $C$ given by

$$
C:=n_{k} \rightarrow \cdots \rightarrow(n-2)_{k+1} \rightarrow n_{k} .
$$

Proof. Cycles in $G(n, k)$ remain cycles in $G(n+1, k)$ by Lemma 5.2. The last edge in (5.6) is $E(k, n+1)$, and in view of (5.4), the remaining edges in (5.6) are also in $G(n+1, k)$ by Lemma 5.1 , since none of these are $E(1, n)$.

We say that $G(n, k)$ 's tail $T$ in (5.4) will shed $G(n, k)$ 's cycle $C$ in (5.6) if (5.5) gives the tail in $G(n+1, k)$.

Lemma 5.6. If $k-1$ lies in $G(n, k)$ 's tail $T$, then $T$ will shed a cycle.

Proof. The result follows from Lemma 5.5 and Lemma 4.5.

Lemma 5.7 (Cf. Prop. 2.2 in [5]). The graph $G(n+1, k)$ has either one more or one less cycle than $G(n, k)$.

Proof. Let $T$ denote the tail in $G(n, k)$. If $k-1$ is not in $T$, then by Lemma 5.4, $T$ will absorb a cycle, so $G(n+1, k)$ has one less cycle than $G(n, k)$. If $k-1$ lies in $T$, then by Lemma 5.6, $T$ will shed a cycle, so $G(n+1, k)$ has one more cycle than $G(n, k)$. 


\section{Line graph connecting points $(n, N(n, k))$}

For fixed $k$, consider the Cartesian graph with $n$ on the horizontal axis and $N(n, k)$ on the vertical axis. Create a line graph connecting the points $(n, N(n, k))$. Since $G(n, k)$ depends only on the value of $n$ modulo $k^{2}+k$, we restrict our line graph to values of $n$ between 0 and $k^{2}+k$.

In this section, we will show that the union of this line graph with the horizontal axis has the multimodal shape of a chain of adjoining isosceles right triangles whose hypotenuses sit on the horizontal axis. This shape is actually a consequence of a general result of Price et al. [5, Cor. 2.4]. (One could see this by viewing $A(n, k)$ as a matrix over a field of $p$ elements, where $p>k(k+1)$ is prime, and then showing that the nullity with respect to this underlying finite field is the same as $N(n, k)$.) However, instead of appealing directly to [5, Cor. 2.4], we characterize the shape of the line graph using properties of $G(n, k)$, because this enables us in Section 7 to explicitly determine the coordinates of all the local peaks.

The multimodal shape of the line graph is illustrated in Figure 1 for $k=6$, $0 \leq n \leq 42$. Each dot on the horizontal axis indicates a point where the nullity is zero, and each dot at the apex of a triangle indicates a point where the nullity has a local peak.

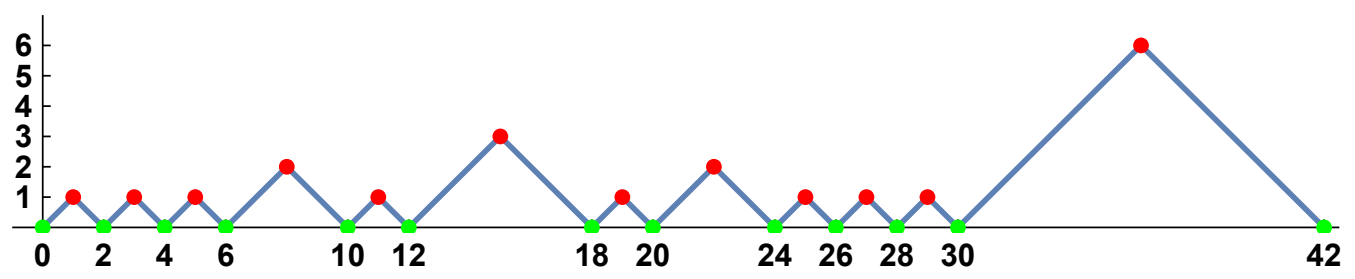

Figure 1: Line graph for $\mathrm{k}=6$

The figure shows that the apex at $n=36$ has the maximum height 6 . In general, $N\left(k^{2}, k\right)=k$, which is the special case $t=k, i=0$ of Theorem 8.7.

When the line graph for $k=6$ is extended for all $n$ on the horizontal axis, one sees the symmetry about the vertical line $n=15$. For general $k$, the symmetry is about $n=\left(k^{2}-k\right) / 2$. This can be explained by the fact that the graph $G(n, k)$ is isomorphic to the graph $G\left(k^{2}-k-n, k\right)$, via the isomorphism that fixes the vertex $k$ and takes every other vertex $a$ in $G(n, k)$ to the vertex $k-1-a$ in $G\left(k^{2}-k-n, k\right)$.

To describe the shape of the line graph for general fixed $k$, we start at 
any point $(n, 0)$ on the horizontal axis where the nullity is zero (for example, the origin). The corresponding graph $G(n, k)$ has no cycles and has a tail $T_{0}$ of length $k$ that begins with a path

$$
B:=k \rightarrow \cdots \rightarrow k-1
$$

since $k-1$ lies in $T_{0}$. Let $q$ denote the length of $B$ and write $k=f q+m$, where $f=[k / q]$ and $0 \leq m<q$. Since $k-1$ is in $T_{0}$, it follows from Lemma 5.6 that $T_{0}$ will shed some cycle $C$ in $G(n+1, k)$. In $G(n+1, k)$, the translate $B+1$ is a cycle containing 0 , which can be seen by replacing $n$ by $n+1$ in all of the edges $E(i, n)$. Since $G(n+1, k)$ has only one cycle, we see that $C=B+1$, so that $C$ is a cycle of length $q$ that contains 0 . The tail $T_{1}$ of $G(n+1, k)$ has length $k-q$. As we continue incrementing the first argument in $G$, we now argue that the process of shedding can continue, so that for each $\omega \in[1, f]$, the graph $G(n+\omega, k)$ has $\omega$ contiguous cycles

$$
C, C+1, \ldots, C+\omega-1
$$

along with a tail $T_{\omega}$ of length $k-\omega q$. (Note that $C-1$ can never be a cycle, since it contains the vertex $k$.) The process of shedding was able to continue as long as $\omega<f$, because then $k-1$ must be in the tail $T_{\omega}$, otherwise $T_{\omega}$ would be shorter than $q$ by Lemma 3.8. The graph $G(n+\omega, k)$ has the cycles in (6.1) by Lemma 4.5 and Lemma 5.2.

For $\omega=f$, the tail $T_{f}$ has length $m<q$, so it is too short to be able to shed a cycle of length $q$. For brevity, write $h=n+f$. Thus, for the graph $G(h, k), k-1$ is in the cycle $C+f-1$ rather than in the tail $T_{f}$. Therefore, by Lemma 3.8 and Lemma 4.2 with $n=h$, the cycles $C$ and $C+f-1$ in $G(h, k)$ are $C^{\prime \prime}$ and $C^{\prime}$, respectively, where $C^{\prime \prime}$ contains both 0 and $(h-1)_{k+1}$, and $C^{\prime}$ contains both $k-1$ and $(h-3)_{k+1}$.

We have so far shown that

$$
N(n+\omega, k)=\omega, \quad 0 \leq \omega \leq f
$$

The points

$$
(n+\omega, N(n+\omega, k))=(n+\omega, \omega), \quad 0 \leq \omega \leq f
$$

all lie on line segment of slope 1 connecting the lowest point $(n, 0)$ to the highest point $(h, f)$. This line segment turns out to be the left leg of an isosceles right triangle, as we now show. 
The graph $G(h, k)$ has $f$ cycles, i.e. $N(h, k)=f$. It remains to prove that

$$
N(h+\omega, k)=f-\omega, \quad 1 \leq \omega \leq f,
$$

which will show that the points

$$
(h+\omega, N(h+\omega, k))=(h+\omega, f-\omega), \quad 0 \leq \omega \leq f
$$

all lie on the right leg of the isosceles right triangle, with the highest point $(h, f)$ situated at the apex and the lowest point $(h+f, 0)$ situated on the horizontal axis.

We proceed to prove (6.3) by showing that as one continues to increment the first argument of $G$, the $f$ cycles of $G(h, k)$ will get absorbed one by one until there are no cycles left. The cycle $C^{\prime \prime}$ in $G(h, k)$ contains $(h-1)_{k+1}$, so it must also contain $h_{k}$, since

$$
E(1, h):=(h-1)_{k+1} \rightarrow h_{k} .
$$

Thus by Lemmas 5.3 and 5.4 , the tail $T_{f}$ in $G(h, k)$ will absorb the cycle $C^{\prime \prime}$. Now $G(h+1, k)$ consists of a tail $T_{f+1}$ of length $m+q$ together with the $f-1$ contiguous cycles

$$
C^{\prime \prime}+1, C^{\prime \prime}+2, \ldots, C^{\prime \prime}+f-1
$$

Recall that $C^{\prime \prime}+f-1$ is the same as the cycle $C^{\prime}$, which contains $k-1$. As the process of absorption continues, $C^{\prime}$ has to be the last cycle absorbed, i.e., $C^{\prime}$ cannot be absorbed while there are still other cycles remaining. This is because once the tail contains both 0 and $k-1$, there can be no cycles left, by Lemma 3.7. By Lemma 5.4, the process of absorption continues as long as $(k-1)$ is not in the tail. Thus, for $1 \leq \omega \leq f$, the graph $G(h+\omega, k)$ will have $f-\omega$ contiguous cycles

$$
C^{\prime \prime}+\omega, \ldots, C^{\prime \prime}+f-1
$$

along with a tail $T_{f+\omega}$ of length $m+\omega q$. In particular, $G(h+f, k)$ has a tail of length $k$ and no cycles. This completes the proof of $(\underline{6.3})$. 


\section{Determination of the nullities $N(n, k)$}

If we knew the coordinates of the apex of a triangle in the line graph, we would know the coordinates of all points on the legs of the triangle. For example, when $k=50$, there is an apex at point $(878,4)$, signifying that when $n=878$, the nullity is 4 , a local peak. The line graph then tells us that as $n$ ranges in order from 874 to 882 , the nullities are $0,1,2,3,4,3,2,1,0$, respectively. Thus, to determine all the nullities, it remains to determine the coordinates of the apexes. This will be accomplished in Theorem 7.5.

For an inequality $Q$, define $\chi(Q)$ to be 1 or 0 according as $Q$ is true or false. We begin with three technical lemmas evaluating the sizes of certain sets of integers $a$. In these lemmas, $M, j, q$, and $y$ are positive integers. Throughout the sequel, for an integer $\ell$, let $(\ell)_{q}$ denote the least nonnegative residue of $\ell$ modulo $q$.

Lemma 7.1. Let $M, j \in[1, q]$ and $y \in[j, q]$. Then

$$
\left|\left\{a \in[0, M-1]: y-j \leq(a j)_{q}<y\right\}\right|=[M j / q]+\chi\left(y \leq(M j)_{q}\right) .
$$

Proof. The result is easily checked for $M=1$, so assume as induction hypothesis that (17.1) holds for some $M \in[1, q)$. Then

$$
\begin{aligned}
& \left|\left\{a \in[0, M]: y-j \leq(a j)_{q}<y\right\}\right| \\
& =[M j / q]+\chi\left(y \leq(M j)_{q}\right)+\chi\left(y-j \leq(M j)_{q}<y\right) \\
& =[M j / q]+\chi\left(y-j \leq(M j)_{q}\right) .
\end{aligned}
$$

To complete the induction, it remains to prove that

$$
[M j / q]+\chi\left(y-j \leq(M j)_{q}\right)=[(M+1) j / q]+\chi\left(y \leq((M+1) j)_{q}\right) .
$$

Since $x=q[x / q]+x_{q}$ for any positive integer $x$, we have

$$
M j=\alpha q+\beta, \text { with } \alpha=[M j / q], \quad \beta=(M j)_{q}
$$

and

$$
((M+1) j)_{q}=(\beta+j)_{q}=(\beta+j)-q[(\beta+j) / q] .
$$

By (7.3) and (7.4), we can write (7.2) in the form

$$
\alpha+\chi(y \leq \beta+j)=\alpha+[(\beta+j) / q]+\chi(y \leq(\beta+j)-q[(\beta+j) / q])
$$


When $\beta+j<q$, both sides of (7.5) match, since $[(\beta+j) / q]=0$. When $\beta+j \geq q$, we have $[(\beta+j) / q]=1$, so that (7.5) becomes

$$
\alpha+1=\alpha+1+\chi(y \leq \beta+j-q) .
$$

Since $y \geq j>\beta+j-q$, the rightmost term in (7.6) vanishes, so that (7.6) holds. This completes the proof of (7.5).

Lemma 7.2. Let $M, j \in[1, q]$ and $y \in[1, j]$. Then

$$
\left|\left\{a \in[0, M-1]: y \leq(a j)_{q}<y+q-j\right\}\right|=M-[M j / q]-\chi\left(y \leq(M j)_{q}\right) .
$$

Proof. Both sides vanish when $j=q$, so we may assume $j<q$. The result is easily checked for $M=1$, so assume as induction hypothesis that (7.7) holds for some $M \in[1, q)$. Then

$$
\begin{aligned}
& \left|\left\{a \in[0, M]: y \leq(a j)_{q}<y+q-j\right\}\right| \\
& =M-[M j / q]-\chi\left(y \leq(M j)_{q}\right)+\chi\left(y \leq(M j)_{q}<y+q-j\right) \\
& =M-[M j / q]-\chi\left(y+q-j \leq(M j)_{q}\right) .
\end{aligned}
$$

To complete the induction, it remains to prove that

$$
-[M j / q]-\chi\left(y+q-j \leq(M j)_{q}\right)=1-[(M+1) j / q]-\chi\left(y \leq((M+1) j)_{q}\right) .
$$

The proof of (7.8) is analogous to that of (17.2), so we omit the details.

When $y=0$, (7.7) holds if the rightmost term is omitted, as is shown in the following lemma.

Lemma 7.3. Let $M, j \in[1, q]$. Then

$$
\left|\left\{a \in[0, M-1]: 0 \leq(a j)_{q}<q-j\right\}\right|=M-[M j / q] .
$$

Proof. This follows by induction on $M$, as with the preceding two lemmas.

Given an edge $E:=a \rightarrow b$ in $G(n, k)$, define its length to be $|E|=$ $(b-a)_{k+1}$. There are only two possible edge lengths, as is shown in the next lemma. 
Lemma 7.4. Given an edge $E:=a \rightarrow b$ in $G(n, k)$, its length is

$$
|E|= \begin{cases}\left(n_{k}-n_{k+1}+2\right)_{k+1} & \text { if } \quad b \leq n_{k}-1 \\ \left(n_{k}-n_{k+1}+1\right)_{k+1} & \text { if } \quad b \geq n_{k} .\end{cases}
$$

Proof. For some $i \in[1, k]$ we have

$$
a=(n+i-2)_{k+1}, \quad b=(n+i-1)_{k}, \quad|E|=\left(b-n_{k+1}-i+2\right)_{k+1} .
$$

If $b \leq n_{k}-1$, then $b=n_{k}+i-1-k$, so that

$$
|E|=\left(n_{k}+i-1-k-n_{k+1}-i+2\right)_{k+1}=\left(n_{k}-n_{k+1}+2\right)_{k+1} .
$$

If $b \geq n_{k}$, then $b=n_{k}+i-1$, so that

$$
|E|=\left(n_{k}+i-1-n_{k+1}-i+2\right)_{k+1}=\left(n_{k}-n_{k+1}+1\right)_{k+1} .
$$

In the first case of (17.10), we call $E$ a long edge, while in the second case, we call $E$ a short edge.

Fix $k$. In preparation for Theorem 7.5, we introduce the following notation. Let $q$ be an integer in the interval $[1, k]$. From the division algorithm,

$$
k=f q+m, \text { where } f=f(q):=[k / q], \quad m=m(q):=k_{q} .
$$

Let $j \in[1, q]$ be an integer which is relatively prime to $q$. Define

$$
s(q, j):=\left|\left\{a \in[1, m]:(a j)_{q} \leq(M j)_{q}\right\}\right|, \text { where } M:=m+1 .
$$

For integer $r \in[1, q-1]$, define

$$
t(r, q, j):=\left|\left\{a \in[0, m]:(a j)_{q}<(r j)_{q}\right\}\right| .
$$

Note that

$$
0 \leq s(q, j) \leq m, \quad 1 \leq t(r, q, j) \leq M .
$$

Let $\eta$ denote the specific value of $n$ defined by

$$
\eta=\eta(q, j):=k[(k+1) j / q]-k+(M j)_{q} f+s(q, j) .
$$


It's not difficult to check that $\eta(q, j) \in\left[f, k^{2}+k-f\right]$. For each integer $w \in[1, f]$, define

$$
c(r)=c(r, w, q, j):=-1-w+(r j)_{q} f+t(r, q, j), \quad 1 \leq r \leq q-1,
$$

and set

$$
c(0)=c(q):=k-w .
$$

It is easy to check that for each $w \in[1, f]$, the integers $c(1), \ldots, c(q-1)$ are distinct and lie in the interval $[0, k-f-1]$.

Reducing modulo $k$ and modulo $k+1$ in (7.14), we have

$$
\eta_{k}=(M j)_{q} f+s(q, j)
$$

and

$$
\eta_{k+1} \equiv-[(k+1) j / q]+1+(M j)_{q} f+s(q, j) \quad(\bmod k+1) .
$$

Thus by Lemma 7.4, the long edges in $G(\eta, k)$ have length

$$
\left(\eta_{k}-\eta_{k+1}+2\right)_{k+1}=\left\{\begin{array}{lll}
1 & \text { if } & q=1 \\
{[(k+1) j / q]+1} & \text { if } & q>1
\end{array}\right.
$$

and the short edges in $G(\eta, k)$ have length

$$
\left(\eta_{k}-\eta_{k+1}+1\right)_{k+1}=\left\{\begin{array}{lll}
0 & \text { if } & q=1 \\
{[(k+1) j / q]} & \text { if } & q>1 .
\end{array}\right.
$$

Observe that

$$
[(k+1) j / q]=f j+[M j / q] .
$$

We are now prepared to identify the coordinates of the apexes in our line graph.

Theorem 7.5. The graph $G(\eta(q, j), k)$ has $f$ distinct cycles of length $q$ given by

$$
k-w=c(0) \rightarrow c(1) \rightarrow \cdots \rightarrow c(q)=k-w, \quad w \in[1, f],
$$

so that the line graph has a local peak at each point in the set

$$
\mathcal{S}:=\{(\eta(q, j), f): 1 \leq q \leq k, 1 \leq j \leq q, \operatorname{gcd}(j, q)=1\} .
$$

Moreover, the points in $\mathcal{S}$ comprise the totality of local peaks in our line graph. 
Proof. We begin by proving that for each $r \in[1, q-1]$,

$$
c(r) \leq \eta_{k}-1 \quad \text { if and only if }(r j)_{q} \leq(M j)_{q} .
$$

First suppose that $(r j)_{q} \leq(M j)_{q}$. Subtracting (7.15) from (7.17), we have

$$
\begin{aligned}
& \eta_{k}-c(r)= \\
& \left((M j)_{q}-(r j)_{q}\right) f+w+\left|\left\{a \in[1, m]:(r j)_{q} \leq(a j)_{q} \leq(M j)_{q}\right\}\right| .
\end{aligned}
$$

The right side of (17.24) is greater or equal to $0+w+0=w \geq 1$; thus (7.23) holds in this case.

Next suppose that $(r j)_{q}>(M j)_{q}$. Subtracting (7.17) from (17.15), we have

$$
\begin{aligned}
& c(r)-\eta_{k}= \\
& \left((r j)_{q}-(M j)_{q}\right) f-w+\left|\left\{a \in[1, m]:(M j)_{q}<(a j)_{q}<(r j)_{q}\right\}\right| .
\end{aligned}
$$

The right side of $(7.25)$ is greater or equal to $f-w+0 \geq 0$. This completes the proof of (7.23).

For $r=q$, (7.23) fails to hold; instead we have

$$
c(q)=k-w \geq \eta_{k} .
$$

This follows because

$$
w+\eta_{k} \leq f+\eta_{k}=\left(1+(M j)_{q}\right) f+s(q, j) \leq q f+m=k .
$$

We proceed to prove that $(7.22)$ is a cycle in $G(\eta, k)$ for each $w \in[1, f]$. First consider the case $q=1$, wherein $f=k, m=0$, and $\eta=k^{2}$. By (2.2), all $k$ edges in $G\left(k^{2}, k\right)$ are loops of the form $(i-1)_{k+1} \rightarrow(i-1)_{k+1}$. Thus, when $q=1$, (17.22) is a cycle in $G(\eta, k)$ (of length 1 ) for each $w \in[1, f]$.

Now assume that $q>1$. By (17.26) and Lemma 7.4, an edge in $G(\eta, k)$ ending with $c(q)=k-w$ must be a short edge. Therefore, in order to conclude that $c(q-1) \rightarrow c(q)$ is an edge in $G(\eta, k)$, it suffices to show its edge length is given by (7.21), i.e., it suffices to show that

$$
(c(q)-c(q-1))_{k+1}=f j+[M j / q] .
$$

The left member of (17.27) equals

$$
\begin{aligned}
& (k-w+1+w+(j-q) f-t(q-1, q, j))_{k+1}= \\
& \left((j-q) f-\left|\left\{a \in[0, m]:(a j)_{q}<q-j\right\}\right|\right)_{k+1}= \\
& (j f+M-(M-[M j / q]))_{k+1}=f j+[M j / q],
\end{aligned}
$$


where the penultimate equality follows from Lemma 7.3. This completes the proof of (7.27).

To prove that (7.22) is a cycle in $G(\eta, k)$, it remains to show that $E_{r}:=$ $c(r-1) \rightarrow c(r)$ is an edge in $G(\eta, k)$ for each $r \in[1, q-1]$. To this end, it suffices to show, in view of (7.23) and Lemma 7.4, that

$$
\left|E_{r}\right|:=(c(r)-c(r-1))_{k+1}=j f+[M j / q]+\chi\left((r j)_{q} \leq(M j)_{q}\right)
$$

for each $r \in[1, q-1]$.

Case 1: $(r j)_{q}>((r-1) j)_{q}$.

We have

$$
\left|E_{r}\right|=\left((r j)_{q}-((r-1) j)_{q}\right) f+\left|\left\{a \in[0, m]:((r-1) j)_{q} \leq(a j)_{q}<(r j)_{q}\right\}\right| .
$$

In Case 1,

$$
((r-1) j)_{q}=(r j)_{q}-j
$$

so that

$$
\left|E_{r}\right|=j f+\left|\left\{a \in[0, m]:(r j)_{q}-j \leq(a j)_{q}<(r j)_{q}\right\}\right| .
$$

Applying Lemma 7.1 with $y=(r j)_{q}$, we deduce (7.28).

Case 2: $(r j)_{q}<((r-1) j)_{q}$.

Since

$$
(c(r)-c(r-1))_{k+1}=k+1-(c(r-1)-c(r))_{k+1},
$$

we have

$\left|E_{r}\right|=k+1+\left((r j)_{q}-((r-1) j)_{q}\right) f-\left|\left\{a \in[0, m]:(r j)_{q} \leq(a j)_{q}<\left((r-1)_{j}\right)_{q}\right\}\right|$.

In Case 2,

$$
((r-1) j)_{q}=(r j)_{q}+q-j,
$$

so that

$$
\left|E_{r}\right|=j f+M-\left|\left\{a \in[0, m]:(r j)_{q} \leq(a j)_{q}<(r j)_{q}+q-j\right\}\right| .
$$

Applying Lemma 7.2 with $y=(r j)_{q}$, we deduce (7.28).

The remark below (7.16) shows that the cycles in (7.22) are distinct. Thus $G(\eta(q, j), k)$ has $f$ cycles of length $q$, which proves that every point in $\mathcal{S}$ is a local peak. 
We proceed to prove that the integers

$$
\eta(q, j) \text { with } 1 \leq q \leq k, \quad 1 \leq j \leq q, \quad \operatorname{gcd}(j, q)=1
$$

are all distinct, so that

$$
|\mathcal{S}|=\sum_{q=1}^{k} \phi(q),
$$

where $\phi$ is Euler's totient function. Suppose that $\eta(q, j)=\eta\left(q^{\prime}, j^{\prime}\right)$. Then $G(\eta(q, j), k)=G\left(\eta\left(q^{\prime}, j^{\prime}\right), k\right)$. Since the cycles in $G(\eta(q, j), k)$ have length $q$ for each $j$, we must have $q=q^{\prime}$. Using (7.19) or (7.20) to compare edge lengths, we have $[(k+1) j / q]=\left[(k+1) j^{\prime} / q\right]$, which forces $j=j^{\prime}$. This completes the proof of (7.29) .

Each point $(\eta(q, j), f) \in \mathcal{S}$ is an apex of an isosceles right triangle in our line graph whose base has length $2 f=2[k / q]$. For each $q \in[1, k]$, there are $\phi(q)$ values of $j$ for which the base of the corresponding triangle has length $2[k / q]$. Summing the base lengths of all the triangles with apexes in $\mathcal{S}$ yields the total length

$$
2 \sum_{q=1}^{k} \phi(q)[k / q] .
$$

Amazingly, this sum equals $k^{2}+k$ [7, Ex. 9, p. 29]. Thus there is no room in the line graph for a triangle whose apex is not in $\mathcal{S}$. This completes the proof that the points in $\mathcal{S}$ comprise the totality of local peaks in our line graph.

\section{Applications of the formulas for $N(n, k)$}

For any fixed nonnegative integer $z$, let $P_{z}(k)$ denote the percentage of matrices $A(n, k)$ with $n \in\left[0, k^{2}+k\right)$ for which $A(n, k)$ has nullity $z$. Theorems 8.1 and 8.2 provide asymptotic formulas for $P_{z}(k)$ as $k \rightarrow \infty$. Theorem 8.1 starts off with the case $z=0$.

Theorem 8.1. Let $P_{0}(k)$ denote the percentage of matrices $A(n, k)$ which are nonsingular. As $k$ gets large, $P_{0}(k)$ approaches $3 / \pi^{2} \simeq 30.4 \%$. 
Proof. For each fixed $k$ and $n \in\left[0, k^{2}+k\right), G(n, k)$ has no cycles if and only if $n$ is the left endpoint of the hypotenuse of a triangle in the line graph. Since there are $|\mathcal{S}|$ triangles in the line graph, it follows that as $k$ gets large, $P_{0}(k)$ is asymptotic to $|\mathcal{S}| /\left(k^{2}+k\right)$. By (7.29) and [7, p. 26], $|\mathcal{S}|$ is asymptotic to $3 k^{2} / \pi^{2}$. Thus $P_{0}(k)$ approaches $3 / \pi^{2}$.

Theorem 8.2. Fix an integer $z \geq 1$. Let $P_{z}(k)$ denote the percentage of matrices $A(n, k)$ which have nullity $z$. As $k$ gets large, $P_{z}(k)$ approaches $3\left(1 / z^{2}+1 /(z+1)^{2}\right) / \pi^{2}$.

Proof. We argue as in the previous proof. The number of triangles of height $z$ in the line graph is

$$
\sum_{k /(z+1)<q \leq k / z} \phi(q)
$$

and each of these triangles contains a single point of height $z$, namely the apex. The number of triangles of height $>z$ is

$$
\sum_{1 \leq q \leq k /(z+1)} \phi(q)
$$

and each of these triangles contains two points of height $z$. Thus there are

$$
\sum_{k /(z+1)<q \leq k / z} \phi(q)+2 \sum_{1 \leq q \leq k /(z+1)} \phi(q)=\sum_{q=1}^{k / z} \phi(q)+\sum_{q=1}^{k /(z+1)} \phi(q)
$$

points of height $z$ in the line graph. The right side is asymptotic to

$$
\frac{3 k^{2}}{z^{2} \pi^{2}}+\frac{3 k^{2}}{(z+1)^{2} \pi^{2}}
$$

so $P_{z}(k)$ approaches $3\left(1 / z^{2}+1 /(z+1)^{2}\right) / \pi^{2}$.

For $k=300$, the exact number of $n \in\left[0, k^{2}+k\right)$ for which $N(n, k)=0$, $N(n, k)=1$, and $N(n, k)=2$ is 27398,34256 , and 9902, respectively. For comparison, the estimates in Theorems 8.1 and 8.2 give

$$
90300 * \frac{3}{\pi^{2}} \simeq 27447.9, \quad 90300 * \frac{15}{4 \pi^{2}} \simeq 34309.9, \quad 90300 * \frac{13}{12 \pi^{2}} \simeq 9911.7 .
$$

Fix $k$. It is not a straightforward task to evaluate $N(n, k)$ for a random value of $n$, since the nullity in Theorem 7.5 is not expressed explicitly as a 
function of $n$. However, when $n$ is given as a function of $k$, we can often apply Theorem 7.5 directly to evaluate $N(n, k)$. The next eight theorems give illustrative examples.

By [2, Lemma 1], $N(2 k-\theta, k)=1$ for all odd $\theta$ with $k-1 \geq \theta \geq-1$. Lemma 9.1 below shows that for all even $\epsilon$ with $k-1 \geq \epsilon \geq-2$, we have

$$
N(2 k-\epsilon, k)=\left\{\begin{array}{llll}
2 & \text { if } & \epsilon \equiv k+1 & (\bmod 3) \\
0 & \text { if } \quad \epsilon \neq k+1 & (\bmod 3) .
\end{array}\right.
$$

A different method is needed to evaluate $N(2 k+b, k)$ when $b \geq 3$. In the next four theorems, we apply Theorem 7.5 to evaluate $N(2 k+b, k)$ for $b \in\{3,4,5,6\}$.

Theorem 8.3. $N(2 k+3, k)$ equals 3 or 1 according as $3 \mid k$ or $3 \nmid k$.

Proof. First suppose that $3 \mid k$. Then by (17.14) with $q=k / 3$ and $j=1$, we have $\eta=2 k+3$, since $m=0$ and $f=3$ when $q=k / 3$. By Theorem 7.5 , $N(2 k+3, k)=N(\eta, k)=f=3$, as desired. Next suppose that $3 \nmid k$. Then with $q=k$ and $j=3$, we have $\eta=2 k+3$, since $m=0$ and $f=1$ when $q=k$. By Theorem 7.5, $N(2 k+3, k)=N(\eta, k)=f=1$, as desired.

Theorem 8.4. $N(2 k+4, k)$ equals 2 or 0 according as $3 \mid k$ or $3 \nmid k$.

Proof. In the proof of Theorem 8.3, the point $(\eta, N(\eta, k))=(2 k+3, f)$ is an apex of a triangle in the line graph, where $f$ is 3 or 1 according as $3 \mid k$ or $3 \nmid k$. The neighboring point to the right of the apex on the line graph is $(2 k+4, f-1)$, so that $N(2 k+4, k)$ equals 2 or 0 according as $3 \mid k$ or $3 \nmid k$.

Theorem 8.5. $N(2 k+5, k)=1$ for all $k \geq 1$.

Proof. The proof of Theorem 8.3 yields the apex $(2 k+3,3)$ or $(2 k+3,1)$ according as $3 \mid k$ or $3 \nmid k$. Moving two units to the right along the line graph yields the point $(2 k+5,1)$ in either case. Thus $N(2 k+5, k)=1$.

Theorem 8.6. For $k>2, N(2 k+6, k)$ equals 2 or 0 according as $k \equiv 1$ $(\bmod 3)$ or $k \not \equiv 1(\bmod 3)$.

Proof. First suppose that $3 \mid k$. As was noted in the proof of Theorem 8.4, the point $(2 k+3,3)$ is an apex of a triangle in the line graph. Moving 
down the right leg of this triangle, we see that $(2 k+6,0)$ is on its base, so $N(2 k+6, k)=0$.

Next suppose that $k \equiv 1(\bmod 3)$. To show that $(2 k+6,2)$ is on the line graph, it suffices to show that $(2 k+7,3)$ is an apex of a triangle. By (7.14) with $q=(k-1) / 3$ and $j=1$, we have $\eta=2 k+7$, since $m=1$ and $f=3$ when $q=(k-1) / 3$. Thus by Theorem $7.5,(\eta, f)=(2 k+7,3)$ is an apex.

Finally suppose that $k \equiv 2(\bmod 3)$. This is the trickiest case. To show that $(2 k+6,0)$ is on the line graph, it suffices to show that $(2 k+5,1)$ is an apex of a triangle. From (7.14) with $q=(2 k-1) / 3$ and $j=2$, we can prove that $\eta=2 k+5$, using the facts that $m=(k+1) / 3$ and $f=1$ when $q=(2 k-1) / 3$. The proof that $\eta=2 k+5$ is facilitated by the observations that $(2 M)_{q}=3$ and $s(q, 2)=2$, where the last equality follows because $a=1$ and $a=m$ are the only values of $a$ satisfying the inequality in (7.12). Thus by Theorem $7.5,(\eta, f)=(2 k+5,1)$ is an apex.

Theorem 8.7. Let $t, k \geq 2$ and suppose that $(t+1) \mid(k+1)$. Then

$$
N(t k+i, k)=N(t k-i, k)=t-i, \quad 0 \leq i \leq t .
$$

Proof. By (7.14) with $q=(k+1) /(t+1)$ and $j=1$, we have $\eta=t k$, since $m=(k-t) /(t+1)$ and $f=t$ when $q=(k+1) /(t+1)$. Thus by Theorem 7.5, $(\eta, f)=(t k, t)$ is an apex, which proves the result for $i=0$. For general $i \in[0, t]$, the result follows by descending from the apex along the line graph.

Theorem 8.8. For $1<c<k$, we have $N(c k+c-k, k)=\operatorname{gcd}(c, k)$.

Proof. Write $d=\operatorname{gcd}(c, k)$. By (7.14) with $q=k / d$ and $j=c / d$, we have $\eta=c k+c-k$, since $m=0$ and $f=d$ when $q=k / d$. Thus by Theorem 7.5 , $(\eta, f)=(c k+c-k, d)$ is an apex, and $N(c k+c-k, k)=d$.

Theorem 8.9. When $k$ is even, $A(3 k, k)$ is nonsingular, i.e., $N(3 k, k)=0$. When $k$ is odd, $N(3 k, k)$ equals 1 or 3 according as $k \equiv 1(\bmod 4)$ or $k \equiv 3$ $(\bmod 4)$.

Proof. First suppose that $k \equiv 3(\bmod 4)$. Then $N(3 k, k)=3$ by the special case $t=3, i=0$ of Theorem 8.7.

Next suppose that $k \equiv 1(\bmod 4)$. By (7.14) with $q=(k+1) / 2$ and $j=2$, we have $\eta=3 k$, since $m=(k-1) / 2$ and $f=1$ when $q=(k+1) / 2$. Thus by Theorem $7.5,(\eta, f)=(3 k, 1)$ is an apex, and $N(3 k, k)=1$. 
Next suppose that $k \equiv 0(\bmod 4)$. By (17.14) with $q=k / 4$ and $j=1$, we have $\eta=3 k+4$, since $m=0$ and $f=4$ when $q=k / 4$. Thus by Theorem $7.5,(\eta, f)=(3 k+4,4)$ is an apex of a triangle, so that $N(3 k+4, k)=4$. Moving four units to the left of the apex, we obtain the point $(3 k, 0)$ on the base of the triangle, so that $N(3 k, k)=0$.

Finally suppose that $k \equiv 2(\bmod 4)$. It is straightforward to check that $A(6,2)$ is nonsingular, so we may assume that $k \geq 6$. By (7.14) with $q=$ $(k+2) / 4$ and $j=1$, we have $\eta=3 k-3$, since $m=(k-6) / 4$ and $f=3$ when $q=(k+2) / 4$. Thus by Theorem $7.5,(\eta, f)=(3 k-3,3)$ is an apex of a triangle, so that $N(3 k-3, k)=3$. Moving three units to the right of the apex, we obtain the point $(3 k, 0)$ on the base of the triangle, so that $N(3 k, k)=0$.

Theorem 8.10. Let $a, b$ be integers with $b \geq 1$ and $0 \leq a \leq b$. Then

$$
N((k+1+a-b)(k-a) / b, k)=(k-a) / b, \quad \text { if } k \equiv a \quad(\bmod b) .
$$

Proof. First suppose that $a<b$. Then by (7.14) with $b=q$ and $j=1$, we have $\eta=(k+1+a-b)(k-a) / b$, since $m=a$ and $f=(k-a) / b$. Since $(\eta, f)$ is an apex by Theorem 7.5, the result follows in this case. Now suppose that $a=b$. We must prove that

$$
N(-1-k+(k+1) k / b, k)=-1+k / b .
$$

By (7.14) with $b=q$ and $j=1$, we have $\eta=-k+(k+1) k / b$, since $m=0$ and $f=k / b$. Since $(-k+(k+1) k / b, k / b)$ is an apex by Theorem 7.5 , the result follows by moving one unit to the left of the apex along the line graph.

For example, take $b=4$. Then we have the nullity formulas

$$
\begin{aligned}
& N\left(\left(k^{2}-3 k\right) / 4, k\right)=k / 4, \quad \text { if } k \equiv 0 \quad(\bmod 4) \text {, } \\
& N\left(\left(k^{2}-3 k+2\right) / 4, k\right)=(k-1) / 4, \quad \text { if } k \equiv 1 \quad(\bmod 4) \text {, } \\
& N\left(\left(k^{2}-3 k+2\right) / 4, k\right)=(k-2) / 4, \quad \text { if } k \equiv 2 \quad(\bmod 4) \text {, } \\
& N\left(\left(k^{2}-3 k\right) / 4, k\right)=(k-3) / 4, \quad \text { if } k \equiv 3 \quad(\bmod 4) .
\end{aligned}
$$




\section{Relation between $D(n, k, x)$ and $N(n, k)$}

Recall from the spectral theorem [4, Theorem 12.2.2] that $A(n, k)$ is unitarily diagonalizable. In particular, its nonzero eigenvalues are purely imaginary and occur in complex conjugate pairs, so that $A(n, k)$ has even rank.

In the Introduction, we conjectured that for even $n$, the zero $x=0$ of the polynomial $D(n, k, x)$ has multiplicity $N(n, k)$, where $D(n, k, x)$ is the determinant of the payoff matrix $A(n, k, x)$. Since $A(n, k)$ is diagonalizable, the conjecture can be restated as follows: for even $n$, the multiplicity of the zero $x=0$ of the determinant of $A(n, k, x)$ equals the multiplicity of the 0 eigenvalue of $A(n, k, 0)$. Using Mathematica, we have verified this conjecture for all even $n$ with $n \leq 150$.

The conjecture is easily proved for example when $k=1$, but it is open for general $k<(n-2) / 2$. In Theorem 9.2 , we prove the conjecture for all $k \geq(n-2) / 2$. Let $\nu:=n / 2 \in \mathbb{Z}$ and assume throughout the remainder of this section that $2 \nu-1 \geq k \geq \nu-1 \geq 1$.

Our matrix $A(n, k, x)$ is the same as the matrix $M(n, n-k-1, x)$ defined in [3]. Thus, by [3, Lemma 2.3], we have

$$
D(2 \nu, k, x)=F_{2 \nu-k-1}^{2}(x),
$$

where for integers $a \geq 0$,

$$
F_{0}(x)=1, F_{1}(x)=x, \quad F_{a+2}(x)=(x+1) F_{a+1}(x)-F_{a}(x) .
$$

Induction on $a$ then shows that

$$
F_{3 a}(0)=(-1)^{a}, \quad F_{3 a+1}(0)=0, \quad F_{3 a+2}(0)=(-1)^{a+1},
$$

and for the derivatives,

$$
F_{3 a}^{\prime}(0)=(-1)^{a} a, F_{3 a+1}^{\prime}(0)=(-1)^{a}(2 a+1), F_{3 a+2}^{\prime}(0)=(-1)^{a}(a+1) .
$$

Lemma 9.1. Suppose that $2 \nu-1 \geq k \geq \nu-1 \geq 1$. Then $N(2 \nu, k)=2$ if $k \equiv 2 \nu+1(\bmod 3)$, and $N(2 \nu, k)=0$ otherwise.

Proof. By (9.1) and (9.3), $A(2 \nu, k)$ is singular if and only if $k \equiv 2 \nu+1$ (mod 3). Suppose that this congruence holds. Since $A(2 \nu, k)$ is a singular skew-symmetric matrix with even rank, its nullity must be at least 2 . We must prove that the nullity is exactly 2 . Appealing to [3, Lemma 2.1], we see that $A(2 \nu-1, k)$ has nullity 1 when $k<2 \nu-1$, and $A(2 \nu+1, k)$ has nullity 1 when $k=2 \nu-1$. Thus by Lemma 5.7 , the nullity of $A(2 \nu, k)$ cannot be higher than 2 , so it is exactly 2 . 
Theorem 9.2. Suppose that $2 \nu-1 \geq k \geq \nu-1 \geq 1$. Then the zero $x=0$ of $D(2 \nu, k, x)$ has multiplicity $N(2 \nu, k)$.

Proof. By (9.1) and (9.3), $D(2 \nu, k, 0)$ is nonzero if and only if $k \not \equiv 2 \nu+1$ $(\bmod 3)$. First suppose that $k \neq \equiv 2 \nu+1(\bmod 3)$. Then the multiplicity of the zero $x=0$ in $D(2 \nu, k, x)$ is zero. By Lemma 9.1, $N(2 \nu, k)=0$.

Next suppose that $k \equiv 2 \nu+1(\bmod 3)$, so that the multiplicity of the zero $x=0$ in $D(2 \nu, k, x)$ is at least 1. By Lemma 9.1, $N(2 \nu, k)=2$, so in view of (9.1), it remains to show that the coefficient of $x$ in the polynomial $F_{2 \nu-k-1}(x)$ is nonzero. This follows from the formula for $F_{3 a+1}^{\prime}(0)$ in (9.4).

\section{References}

[1] R. J. Evans and G. A. Heuer, Silverman's game on discrete sets, Linear Algebra and its Applications 166 (1992), 217-235.

[2] R. J. Evans and Nolan Wallach, Pfaffians and strategies for integer choice games, Harmonic Analysis, Group Representations, Automorphic Forms and Invariant Theory, Edited by Li, Tan, Wallach, Zhu, Lecture Notes Series, Institute for Mathematical Sciences, National University of Singapore, World Scientific, 2007, pp. 53-72.

[3] R. J. Evans and Nolan Wallach, Pfaffians for Toeplitz payoff matrices, Linear Algebra and its Applications 577 (2019), 114-120.

[4] S. R. Garcia and R. A. Horn, A second course in linear algebra, Cambridge University Press, 2017.

[5] G. L. Price and G. H. Truitt, On the ranks of Toeplitz matrices over finite fields, Linear Algebra and its Applications 294 (1999), 49-66.

[6] G. L. Price and M. Wortham, On sequences of Toeplitz matrices over finite fields, Linear Algebra and its Applications 561 (2019), 63-80. https://arxiv.org/abs/1804.00983

[7] H. E. Rose, A Course in Number Theory, 2nd ed., Clarendon Press, Oxford, 1995.

[8] W. F. Trench, On the eigenvalue problem for Toeplitz band matrices, Linear Algebra and its Applications 64 (1985), 199-214. 